\title{
Archéopages
}

Archéopages

Archéologie et société

34 | 07/2012

Campagnes

\section{À la recherche du champ permanent. Débat}

Propos recueillis par Catherine Chauveau

Cyril Marcigny, Émilie Gauthier et Catherine Chauveau

\section{CpenEdition}

Journals

Édition électronique

URL : https://journals.openedition.org/archeopages/406

DOI : $10.4000 /$ archeopages. 406

ISSN : 2269-9872

Éditeur

INRAP - Institut national de recherches archéologiques préventives

Édition imprimée

Date de publication : 1 février 2012

Pagination : 78-83

ISSN : 1622-8545

Référence électronique

Cyril Marcigny, Émilie Gauthier et Catherine Chauveau, «À la recherche du champ permanent. Débat », Archéopages [En ligne], 34 | 07/2012, mis en ligne le 01 juillet 2012, consulté le 21 janvier 2022. URL : http://journals.openedition.org/archeopages/406 ; DOI : https://doi.org/10.4000/archeopages.406 
À la recherche du champ permanent La palynologie a été liée à l'archéologie dès ses débuts en France. Les recherches limitées d'abord à la Préhistoire se sont aujourd'hui systématisées sur toutes les périodes. On sait à présent, quand on contemple nos paysages actuels, qu'ils sont le résultat de profonds remaniements qui ont commencé il y a près de sept millénaires, dans le but de créer et aménager les espaces agropastoraux si caractéristiques des campagnes actuelles.

\section{Cyril Marcigny}

spécialiste de l'âge du Bronze à l'Inrap et au Centre de recherche en Archéologie, Archéosciences, Histoire, a publié récemment : «Au bord de la mer. Rythmes et natures des occupations protohistoriques en Normandie (III ${ }^{e}$ millénaire-fin de l'âge du Fer)», in M. Honegger et C. Mordant (éd.), Au bord de l'eau, Archéologie des zones littorales du Néolithique à la Protohistoire, $135^{ }$Congrès CTHS (Neuchâtel, Suisse, 2010), Cahier d'Archéologie Romande, 2012, p. 345-364; avec V. Carpentier (dir.), Des Hommes aux Champs I. Pour une archéologie des espaces ruraux du Néolithique au Moyen Âge, Actes de la table ronde de Caen (octobre 2008), Presses Universitaires de Rennes, à paraître; avec L. Leśpez, M. Clet-Pellerin, R. Davidson, B. Mennesson et B. Hardel, «Les paysages de la Hague du Néolithique à nos jours. Le rôle des sociétés dans la construction des paysages d'un finistère atlantique », in L. Lespez (éd.), Paysages et gestion de l'eau, sept millénaires d'histoire de vallées et de plaines littorales en BasseNormandie, hors-série Enquêtes Rurales, Presses Universitaires de Caen-MRSH, 2012, p. 115-142 ; avec B. Van den Bossche, "The pattern of agricultural activities in the Norman countryside (2500-30 BC) as seen through preventive excavations of the south side of Caen », in G. Blancquaert et al., Undersanting the Past: a matter of surface-area, Acts of the XIII' ${ }^{\text {th }}$ Session of the EAA Congress Zadar 2007, BAR International Series 2194, 2011, p. 49-71.

\section{Émilie Gauthier}

maître de conférences à l'université de Franche-Comté et membre du Laboratoire Chrono-environnement, a publié récemment : avec $\mathrm{H}$. Richard, C. Massa, B. Perren, V. Bichet, L. Millet (soumis), «L'agriculture médiévale et contemporaine au Groenland : retro-observation des pratiques et conséquences sur l'environnement », in Étudier les interactions hommesmilieux Questions et réflexions autour des pratiques de la recherche scientifique en environnement, Eds Quae, coll. Indisciplines ; avec E. Doyen, B. Vannière, V. Bichet, H. Richard, Vegetation history and anthropogenic landscape management from 6500 to 1500 cal. BP at Lake Antre (Gallo-roman sanctuary of Villards d'Héria, Jura, France), Vegetation History and Archaeobotany, online 22 May 2012, doi 10.1007/soo334-012-0364-o ; avec A. Laine, H. Richard, «L'époque galloromaine des plaines de Saône au plateaux jurassiens. Bilan des données palynologiques », in M. Reddé et al. (dir.), Aspect de la Romanisation dans l'est de la Gaule, Collection Bibracte21, 1, 2011, p. 329-334; Forêts et agriculteurs du Jura. Les quatre derniers millénaires, Annales Littéraires de l'Université de FrancheComté 765, coll. Environnement, sociétés et archéologie, 6, Presses Universitaires de Franche-Comté, 2004.
Propos recueillis par Catherine Chauveau. Merci à Christiane Descombin 


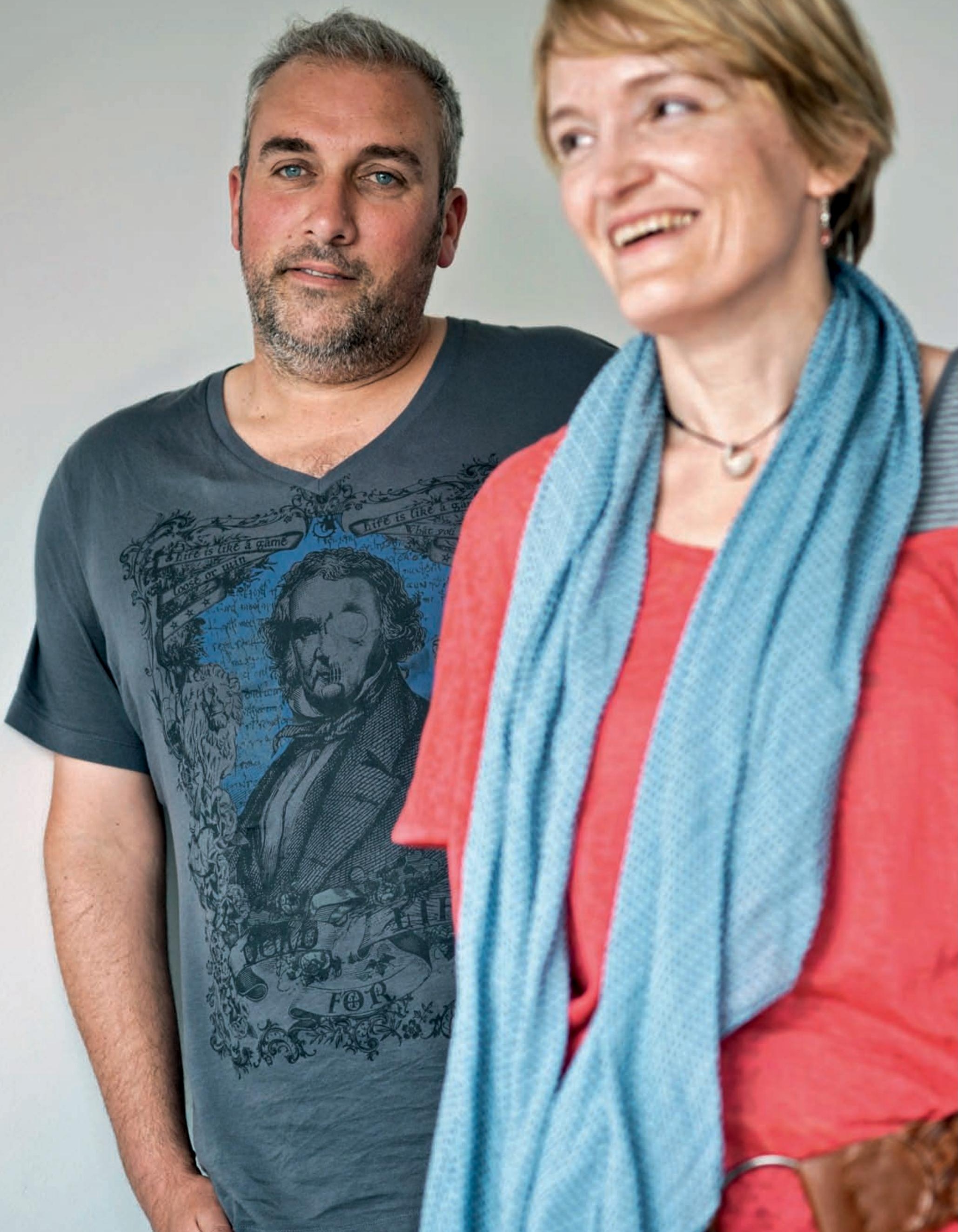


Émilie Gauthier L'attention des palynologues pour l'impact de l'homme sur l'environnement apparaît d'abord en Europe du Nord avec les premières mises en évidence des indices polliniques d'anthropisation. Un peu plus tard, sur le Jura par exemple, il y a les toute premières ébauches de diagrammes polliniques, effectuées juste après guerre par Fridolin Firtion', à partir de 110 sondages dans les tourbières dont le but était d'abord d'évaluer la disponibilité et la rentabilité de cet éventuel combustible. Dans les années 1960, Samuel Wegmüller² étudie plusieurs séquences polliniques holocènes dans sa thèse sur le Jura. L'une des premières à avoir fait de la palynologie en France est Arlette Leroi-Gourhan', et tout de suite en lien très fort avec l'archéologie. Dans les années 1980, Hervé Richard à partir de forages sur les lacs de Chalain ${ }^{\mathbf{4}}$ et de Clairvaux et ainsi que dans des tourbières, a produit plusieurs séquences holocènes qui ont permis de préciser l'histoire de l'évolution de la végétation et des impacts anthropiques. Les palynologues travaillant sur l'évolution de la végétation, n'ont longtemps perçu l'homme que comme un perturbateur brouillant le signal climatique. Or le climat n'est pas le seul à avoir un impact sur la végétation ; forcing climatique et anthropique, souvent étroitement imbriqués, sont souvent difficiles à différencier à partir des paramètres paléoenvironnementaux utilisés. Dans mon laboratoire, j'ai la chance de côtoyer des archéologues, des historiens, des sédimentologues, des géochimistes, la réflexion en commun est plus «globale» et me semble primordiale.

Cyril Marcigny En archéologie protohistorique et historique, la recherche sur les relations homme/milieu n'est devenue systématique qu'avec l'accroissement des fouilles préventives sur grandes surfaces, avec diverses approches selon les périodes étudiées et les écoles. Â partir des travaux pionniers de Gérard Chouquer et François Favory sur la centuriation d'Orange, il y a tout un pan de la recherche qui s'est ouvert sur la résilience des formes du paysage et la genèse des paysages. Il reste encore à rapprocher réellement les données pour voir comment tirer parti des résultats des diverses disciplines.

ÉG Lintérêt est bien là : mettre nos données palynologiques en parallèle avec d'autres paramètres archéologiques, historiques, sédimentologiques, géochimiques... Cela prend plus de temps car il faut d'abord des datations radiocarbone très précises pour obtenir un modèle d'âge qui soit cohérent. La présence de l'homme se traduit, à coup sûr, par l'apparition de plantes qui, venant d'ailleurs et de très loin, n'ont pu être apportées que par lui. Au début du Néolithique, dans l'est de la France, ces plantes sont originaires du Proche-Orient et ce sont des céréales, des messicoles, ${ }^{\mathbf{6}}$ des rudérales ${ }^{7}$. Mais d'autres plantes déjà présentes dans l'environnement ont été favorisées par les activités humaines, les défrichements notamment, qui leur permettent de recevoir plus de lumière et de se développer. Si nous pouvons constater cette diminution du couvert forestier, on ne peut pas toujours écarter qu'elle soit due à une cause naturelle comme une tempête violente. Ce qui a toujours été dit et écrit, c'est que c'est seulement lorsque ces différentes plantes apparaissent en même temps que nous pensons avoir un indice suffisant de présence humaine.

CM Même si l'amélioration des datations radiocarbone a grandement contribué à dater précisément les diagrammes polliniques et les données archéologiques et surtout à les corréler, il subsiste souvent un hiatus : les tout premiers indices polliniques d'anthropisation dans le Jura sont très précoces et antérieurs aux sites rubannés. A t-on affaire à de réelles pratiques agricoles, développées par des autochtones mésolithiques ou par des Néolithiques? Peut-être que le jour où on trouvera un niveau mésolithique avec des grains de céréales, les carpologues auront une réponse.

ÉG Utiliser les datations 14C, cela nous fait au moins un langage commun! Les méthodes actuelles plus sophistiquées et qui permettent de calculer un modèle d'âge à partir de plusieurs dates radiocarbone ont abouti à des datations assez précises.

CM Les indices palynologiques d'anthropisation qui ont été au début mis en évidence pour l'Europe du Nord sont-ils forcément pertinents ailleurs? ÉG Pas forcément! Aujourd'hui, certains palynologues essaient de faire leurs propres indices adaptésau climat, au sol de leur région d'étude... La difficulté est de raisonner sur des sols et des flores modernes ; or ce qui existe aujourd'hui n'existait pas obligatoirement avant l'apparition des champs permanents. Les divers spécialistes ne sont pas d'accord sur la date d'apparition de ceux-ci. En l'absence de l'homme, sous nos latitudes, les forêts prédominent mais elles sont plus ou moins denses et parfois plus clairsemées en plaine. Par exemple, au Néolithique ancien, j'ai seulement $70 \%$ de pollens d'arbres en plaine bourguignonne contre $98 \%$ dans le Jura. Au lac Saint-Point, à plus de 800 mètres d'altitude, j'obtiens au cours du Néolithique et de lâge du Bronze, un signal pollinique discontinu avec une alternance de petits pics de pollens de céréales et de petits pics de pollens de plantain, une plante caractéristique des milieux anthropisés, ces petites augmentations sont accompagnées d'autres indices polliniques d'anthropisation. Si je réduis la maille d'échantillonnage, je retrouve ces petits pics alternés pollen/plantain, avec des courbes de pollens d'arbres très irrégulières. Le total avoisine toujours $90 \%$; ce qui suggère un milieu forestier mais très perturbé par des défrichements récurrents. Cela disparaît vers la fin de l'âge du Bronze : les pollens d'arbres sont moins représentés et leurs courbes sont plus régulières tandis que les courbes des indices d'anthropisation, dont les pollens de céréales et de plantain, sont continues et simultanées. J'interprète cela comme le remplacement d'un système agraire par un autre. L'âge du Bronze est aussi la période à laquelle je vois apparaître un nouveau cortège de plantes venues du Proche-Orient, des plantes plutôt liées aux champs permanents. Donc, à mon avis, à cet endroit, la culture en champs permanents ne débute qu'à lâge du Bronze. Mais Pierre Pétrequin voit l'araire apparaître à la fin du IV millénaire 


\section{Les défrichements néolithiques ne sont pas très visibles en palynologie.}

Émilie Gauthier

1. Contribution à l'étude

paléontologique,

stratigraphique et

physico-chimiques

des tourbières du Jura

français, 1950, université

de Strasbourg, mémoires

du service de la carte

géologique d'Alsace

et Lorraine

2. Über die Spät-

und postglaziale

Vegetationgeschichte des

Südwestlichen Jura, Beitr.

Geobot. Landesaufn., 48,

Schweiz, 1966, $142 \mathrm{p}$.

3. Van Campo M.

Leroi-Gourhan, Arl.,

1956a, «Un paysage

forestier rissien dans

l'Yonne ", Bulletin de

la Société botanique

de France, 103, n ${ }^{\circ}$ 5- 6 ,

p. 285-286. Van Campo M

Leroi-Gourhan, A., 1956b,

«Note préliminaire

à l'étude des pollens

fossiles de différents

niveaux des grottes

d'Arcy-sur-Cure",

Bulletin du Museum,

Les recherches ont

débuté dans les années

1970 sous la direction

de Pierre Pétrequin

5. «Localisation et

extension géographique

des cadastres affichés

à Orange », in M. Clavel-

Lévêque (éd.) Cadastre

et espace rural, Actes

de la table-ronde de

Besançon, Paris, 1983

p. 275-295 ; F. Favory,

Les Paysages de l'Antiquité.

Terres et cadastres

de l'occident romain,

Errance, Paris, 1991,

$243 \mathrm{p}$.

6. Plantes annuelles

à germination

préférentiellement

hivernales habitant

dans les moissons.

7. Plantes qui se

développent sur

$2^{\mathrm{e}}$ série, t. XXVIII, 3 ,

des décombres ou

p. 326-330.

dans les friches. 
à Chalain, ce qui, pour lui est un signe d'un passage à une culture permanente. Je pense qu'elle n'apparaît pas partout au même moment sous la même forme et j'ai plutôt tendance à la voir se généraliser à partir du Bronze final.

CM Je suis de cet avis. Les désaccords de datation tiennent, à mon avis, à la volonté de tirer des généralités de phénomènes observés isolément. Au Néolithique, à l'âge du Bronze, ou à l'âge du Fer, il devait y avoir des situations différentes dans ces territoires qui sont les nôtres, avec des points communs. Dans l'Ouest, les palynologues dateraient l'apparition des champs permanents vers le milieu de l'âge du Fer, alors que les archéologues les voient apparaître à partir du Bronze ancien, au campaniforme. Mais en Angleterre, on a ces mêmes structures de champs dès le $\mathrm{IV}^{\mathrm{e}}$ millénaire. En Basse-Normandie, on connaît plusieurs parcellaires de l'âge du Bronze, des ensembles construits en îlots très refermés couvrant quelques hectares. Pour un palynologue, la présence de céréales est trop faible pour qu'il conclue à l'existence d'une agriculture permanente. Alors que pour nous, les vestiges sont clairs ! Il s'agit de petites parcelles d'en moyenne 110 mètres carrés, une surface labourable à l'araire en une journée, séparées par de profonds fossés talutés (comme le bocage). Selon moi, le développement de ces parcellaires autour de la deuxième partie du Bronze ancien, époque où on bâtit les grands tumuli, est plus lié à un pouvoir politique fort qui verrouille le territoire et modifie radicalement le système foncier quà de nouvelles pratiques agricoles. Quand ce pouvoir politique s'effondre au cours du Bronze moyen, ce mode de délimitation de parcellaire s'étiole jusqu'au Bronze final où il disparaît. Le parcellaire fondé dans le sol revient un tout petit peu au Hallstatt D, à une époque où il y a nouveau des élites qui se mettent en place, et perdure jusqu'à La Tène ancienne. Puis il faut attendre le $\mathrm{III}^{\mathrm{e}}$ siècle avant notre ère pour voir réapparaître de grands domaines, les fermes aristocratiques gauloises, à un moment de profonde mutation sociale et technique. ÉG Je suis tout à fait pour raisonner sans perdre de vue cet aspect socio-économique. Aux v ve et vi siècles, je ne constate de déprise agricole que sur les sites d'altitude alors qu'en plaine, ou dans des zones d'altitude qui sont des zones de passage reconnues, comme à La Chaux d'Arlier dans le Haut Doubs, l'exploitation reste identique. On ne peut donc qu'être sceptique sur les thèses présentant les «grandes invasions » comme des phases de dépeuplement massif, de destructions généralisées. Et on rejoint les archéologues qui penchent pour une restructuration de l'habitat car les vestiges archéologiques de l'époque mérovingienne montrent qu'il y a une occupation persistante. Les montagnes du Jura sont occupées parfois depuis le Néolithique et on y observe une succession de moments d'emprise et de déprise agricoles que le facteur climatique ne suffit pas à expliquer. CM Dès que les populations savent utiliser les dénivelés des plateaux, labourer correctement, enrichir les terrains (avec des algues, par exemple), tous les milieux sont exploités. La palynologie me semble particulièrement intéressante quand on fait de l'intra-site. Par exemple, des échantillons pris dans des fossés de l'âge du Fer ont révélé des nénuphars et des noisetiers, une création végétale car on ne les repère pas dans le cortège environnant. Au-delà d'être une délimitation de parcelles, c'est aussi une façon de créer des ressources complémentaires. ÉG C'est que les résultats sont plus ou moins probants selon la taille et la situation du remplissage. L'analyse palynologique d'un puits, par exemple, est souvent décevante pour un archéologue car, en général, le constat est qu'il est devenu un dépotoir, et l'archéologue le savait déjà ! Si c'est un petit puits, une petite mare, on obtiendra une pluie pollinique concernant quelques mètres autour du site étudié et on aura du mal à faire le lien avec ce qui se passe à plus loin à plusieurs centaines de mètres, voire à des kilomètres. Par contre, si l'on intervient sur un grand lac, celui-ci capte une pluie pollinique beaucoup plus régionale. Plus le lac est grand, plus les apports régionaux et extra régionaux vont dominer dans la séquence et on aura une impression fausse : pour le lac du Bourget, par exemple, on verra un milieu complètement forestier alors que l'on sait qu'il y a des habitats et des champs tout autour. D'une façon générale, il y a toujours une surreprésentation des pollens d'arbres. Les plantes à fleurs pollinisées par les insectes sont très peu perceptibles, et les légumineuses, par exemple, produisent très peu de pollen. Avec une bonne connaissance de la pluie pollinique actuelle, le palynologue peut interpréter louverture du milieu, mais il ne peut décrire exactement un paysage, ni situer où étaient les cultures, les pâtures, et quelles surfaces elles avaient. Certains états de la végétation peuvent lui échapper. L'absence de pollens d'arbre n'implique pas forcément l'absence d'arbres : la présence de taillis ou de jeunes arbres (un chêne ne pollinise quà partir de 30-40 ans) est invisible d'un point de vue pollinique. Les indices polliniques d'anthropisation, selon les taxons utilisés, sont plus ou moins abondants dans les diagrammes polliniques. Certains appartiennent à des plantes qui pollinisent beaucoup, d'autres à des végétaux qui produisent peu de pollen ou dont les pollens sont peu dispersés. En utilisant une large gamme de taxons, on doit réussir à distinguer différents types de systèmes agraires, différentes manières de pratiquer l'agriculture. Et sur le temps long, car ce qui m'intéresse surtout dans un échantillon, c'est ce qu'il y avant et ce qu'il y a après. Bien sûr l'aide d'autres disciplines, comme la sédimentologie qui apporte des informations sur l'érosion des sols, me parait indispensable dans ce cas. CM Cette approche diachronique nous retient aussi. Sur un même site, on a demandé aux palynologues d'étudier les mares et obtenu tout l'environnement du site en diachronie car ces mares creusées au $\mathrm{I}^{\mathrm{er}}$ siècle sont maintenues jusqu'à l'époque médiévale. Cela révèle les épisodes de prédominance d'espaces boisés qu'on peut mettre en parallèle avec des phases d'abandon du site et donc des moments de déprise agricole. Ces échantillons contiennent d'ailleurs d'autres choses que des pollens et tout aussi intéressantes à étudier. 
ÉG Tout à fait ! Il y a des débris de matière organique, de spores de champignons, de cyanobactéries... plein de petits microfossiles que nous n'avons appris à regarder - et à déterminer que depuis quelques années. Dans des séquences lacustres et tourbeuses, si je retrouve beaucoup de spores de champignons coprophiles, cela indique la présence d'excréments de bétail et donc de pâturages à proximité. Repérer d'autres microfossiles comme les algues permet par exemple de mettre en évidence des phases de «pollution» des eaux et de changements dans le système lacustre, liés aux perturbations dans le bassin versant.

CM Les végétaux marqueurs chronologiques sont-ils fiables?

ÉG Le palynologue reste d'autant plus prudent qu'il a toujours à l'esprit le risque de pollution des échantillons. Plus que l'apparition, c'est l'augmentation des valeurs de certains taxons qui constitue souvent un marqueur chronologique dans les diagrammes polliniques. Le chanvre et le seigle(seul pollen de céréale qu'on peut identifier de manière sûre), augmentent à partir du haut Moyen Âge. À la même période, dans l'est de la France, les pourcentages de charme augmentent dans pratiquement tous les diagrammes polliniques, qu'il y ait déprises agricoles ou non. Est-ce lié à la mise en place de haies, une prolifération de taillis, une utilisation différente de l'espace ? Le sarrasin est censé arriver au XIII ${ }^{\mathrm{e}}$ siècle, de l'est de l'Europe, sauf qu'on en retrouve des pollens dans des niveaux du Néolithique moyen en Bretagne! Le physalis a aujourd'hui disparu de nos bois à tel point qu'on l'a longtemps cru apporté d'Amérique, or on en récolte des graines à pleins seaux dans tous les sites lacustres du Néolithique et de l'âge du Bronze. Comme beaucoup d'autres exemples, cette plante a pu être utilisée, oubliée et retrouvée. Phénomène culturel, habitudes alimentaires qui changent, influences d'autres populations, de nouveaux contacts... toutes les situations peuvent être envisagées.

\section{Un pollen de céréale ne fait pas plus un indice anthropique qu'un grain ne fait un champ.}

Cyril Marcigny
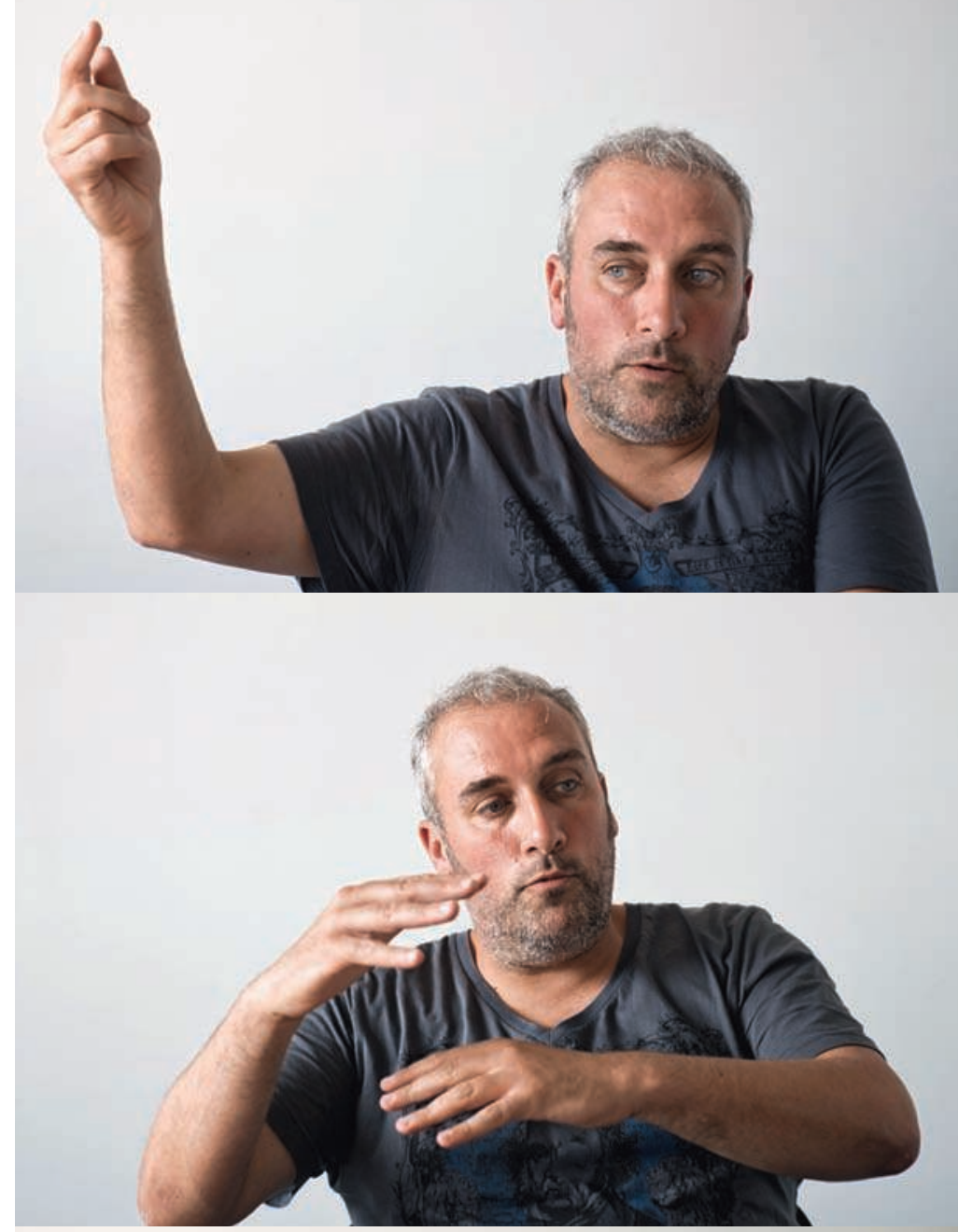